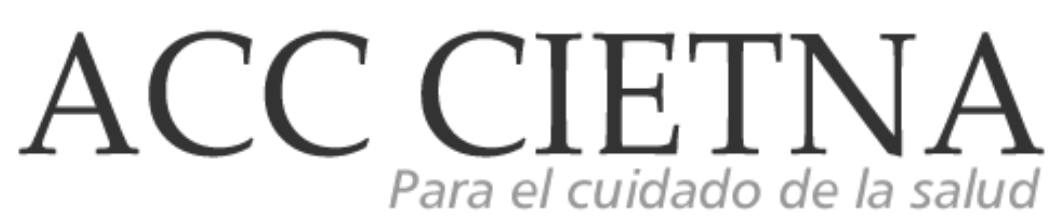

https://doi.org/10.35383/cietna.v1i1.159

\title{
A pesquisa como alicerce da prática baseada em evidências para enfermagem contemporânea: um estudo exploratório
}

\author{
Vieira de Moura, Priscilla ${ }^{1}$, Ortiz Sánchez Maritza Consuelo ${ }^{2}$
}

\section{INFORMACIÓN DEL ARTÍCULO \\ Historia del artículo: \\ Recibido el 23 de mayo de 2016 \\ Aceptado el 15 de junio de 2016}

\section{Palabras claves:}

Enfermagem

Pesquisa

Práticas Baseadas em Evidências

\section{RESUMO}

Objetivo principal: Analisar a visão dos docentes acerca das contribuições das pesquisas para a Prática Baseada em Evidências (PBE) para a enfermagem contemporânea. Metodologia: Estudo descritivo, exploratório, com abordagem qualitativa, tendo como sujeitos doze docentes de enfermagem de uma Instituição Privada de Ensino Superior da Cidade do Rio de Janeiro, Brasil. Resultados principais: Demonstraram que a pesquisa é importante para a mudança da prática de enfermagem baseada em tradições, rituais e tarefas vislumbrando uma prática mais reflexiva alicerçada no conhecimento científico, promovendo assim, a melhoria da qualidade da assistência ao cliente, família e comunidade. Evidenciou-se que a PBE, se encontra incipiente em nosso país em virtude de diversos fatores de ordem pessoal, profissional e institucional. Conclusão principal: Faz-se necessário ampliar o interesse do enfermeiro no desenvolvimento do corpo de conhecimento científico, e das instituições de saúde no sentido de investir no aprimoramento e capacitação dos profissionais, fornecendo o suporte necessário para a realização de pesquisas, visando à melhoria da qualidade dos serviços oferecidos à população, redução de custos, maior reconhecimento e valorização do profissional de enfermagem. Objetivando promover mudanças nas atitudes do enfermeiro frente à pesquisa, esse processo deve ter início na graduação por meio de inclusão de disciplinas curriculares que busquem desenvolver nos estudantes a compreensão da pesquisa para a enfermagem, do processo de pesquisar e da utilização dos resultados de pesquisas para

\footnotetext{
${ }^{1}$ Estudante Centro Universitário da Cidade, Rio de Janeiro, Brasil. Email: pricilavmoura@hotmail.com

${ }^{2}$ Dra en Enfermería, por la Escuela de Enfermería ANNA NERY.Especialista en Gestión y Administración Hospitalaria y Enfermería en el Trabajo, Rio de
} 
a melhoria da assistência ao cliente. Em síntese, para as práticas baseadas em evidências tornar-se realidade, são necessárias mudanças nas esferas educacional, organizacional e individual.

The investigation like foundation of the practice based in evidences for contemporary infirmary: a studio exploratório

ABSTRACT

Keywords:

Nursing

Research

Evidence-based practices
Main Objective: To analyze the views of teachers about the contributions of research for Evidence-Based Practice (EBP) for contemporary nursing. Methodology: A descriptive, exploratory qualitative approach, taking as subjects 12 nursing faculty members of a Private Institution of Higher Education in the City of Rio de Janeiro, Brazil. Main results: These showed that research is important to change nursing practice based on traditions, rituals and tasks, envisioning a more reflective practice grounded in scientific knowledge, thereby promoting, improving the quality of customer, family and community care. It was evident that the EBP is emerging in our country due to many personal, professional and institutional factors. Main conclusion: It is necessary to enlarge the interest of nurses in developing scientific knowledge, and health institutions to invest in upgrading and training of professionals, providing the necessary support for conducting research aimed at improving the quality of services offered to the population, reducing costs, with greater recognition and appreciation of professional nursing. Aiming to promote changes in attitudes of the nurse in the research, this process should begin at the undergraduate through inclusion of curricular disciplines that seek to develop in students an understanding of research in nursing, the process of researching and use of research results to improve customer service. In summary, for evidence-based practices become reality, changes are needed in education, organizational and individual spheres.

\section{Introdução}

Atualmente temos identificado a expansão de estudos sobre as Práticas Baseadas em Evidências (PBE) à nível mundial, agregando não apenas a medicina clínica, mas também outras áreas de saber como a enfermagem. Esta tendência do crescimento leva aos enfermeiros a necessidade da busca pelo conhecimento, realizando pesquisas que proporcionem práticas mais seguras no campo da assistência de enfermagem.
Entende-se que a prática baseada em evidências consiste em um processo de descoberta, avaliação e aplicação de evidências científicas para o tratamento e gerenciamento da saúde. É o cuidado guiado por meio de resultados de pesquisas, consenso de especialistas ou a combinação de ambos'.

A busca e uso de evidências científicas de enfermagem têm como pressuposto utilizar e fomentar a realização de estudos que gerem práticas novadoras de enfermagem, com vista a 
sustentar as ações e as relações do profissional no sistema de saúde, bem como, demonstrar o impacto de tais ações nos resultados do sistema ${ }^{2}$.

Ressalta-se que os profissionais de enfermagem que atuam nas áreas de ensino, assistência e pesquisa precisam somar esforços para demonstrar que os enfermeiros e demais profissionais da equipe de enfermagem, na sua grande maioria no país, não trabalham em quantitativo suficiente, com a qualificação profissional adequada e com os recursos que lhes permitam desenvolver práticas de enfermagem eficazes e eficientes.

No contexto assistencial do país, poucos são os enfermeiros que atuam em ambientes que centram suas ações em evidências científicas. Muitos passam seu dia de trabalho corrigindo falhas no sistema, procurando materiais, trocando equipamentos quebrados, buscando prescrições e documentos deixados em locais errados, corrigindo falhas da lavanderia, farmácia, manutenção, nutrição e limpeza, sendo que, ao final da jornada de trabalho, percebem que não conseguiram realizar cuidados de enfermagem diretos ao paciente, e nem tão pouco, supervisionar de modo eficiente os cuidados prestados por técnicos ou auxiliares de enfermagem ${ }^{2}$.

Dessa forma, o objeto do presente estudo consiste na pesquisa como alicerce da prática baseada em evidências para a Enfermagem contemporânea sob a ótica dos docentes de uma instituição de ensino superior privada do Rio de Janeiro, Brasil.

Nesse contexto, acredita-se na importância da prática do cuidado estar alinhada com resultados de pesquisas proporcionando evidências de maior confiabilidade, além de preencher as lacunas existentes envolvendo a temática PBE e enfermagem, pois mesmo diante do avanço, existe a necessidade de ampliar o desenvolvimento de pesquisas com esse enfoque na enfermagem visando sustentar a prática baseada em evidências.
Assim, a pesquisa baseada em evidências representa um elo entre a pesquisa e a prática profissional - uma ferramenta para capacitação e inserção do profissional melhor capacitado no cotidiano do trabalho, uma vez que permite a aquisição e validação de conhecimentos.

\section{Método}

Estudo descritivo, que consistiu em descrever os dados proporcionados pelos sujeitos do estudo, coerentes com o objeto pesquisado; exploratório, possibilitando uma familiarização com os fatos para uma melhor compreensão dos mesmos; dotado de uma abordagem qualitativa, envolvendo raciocínio indutivo a partir dos discursos elaborados à luz de literaturas pertinentes à temática. É dizer, a pesquisa qualitativa trabalha com o universo de significados, motivos, aspirações, crenças, valores e atitudes. Esse conjunto de fenômenos humanos é entendido aqui como parte da realidade social, pois o ser humano se distingue não só por agir, mas por pensar sobre o que faz e por interpretar suas ações dentro e a partir da realidade vivida e partilhada com seus semelhantes ${ }^{3,4}$.

O processo de trabalho científico foi dividido em três etapas: (1) fase exploratória; (2) trabalho de campo; (3) análise e tratamento do material empírico.

A fase exploratória consistiu na produção do projeto de pesquisa e de todos os procedimentos necessários para a preparação e a entrada em campo, delimitando objeto, objetivo, referências bibliográficas e metodologia 4 .

No trabalho de campo levou-se para a prática empírica a construção teórica elaborada na primeira etapa, desse modo para a coleta dos dados foi utilizado um roteiro de entrevista semiestruturada, com a seguinte pergunta: Quais são as contribuições da pesquisa para a prática 
baseada em evidências na enfermagem contemporânea?. Pergunta em que o entrevistado teve a possibilidade de discorrer sobre o tema em questão ${ }^{4}$.

A entrevista é uma técnica privilegiada de comunicação, em que acontece uma conversa a dois, ou entre vários interlocutores, realizada por iniciativa do entrevistador. No presente estudo ela teve o objetivo de construção de informações pertinentes para o objeto pesquisado 4 .

A terceira etapa envolveu a análise e tratamento do material, realizou-se um conjunto de procedimentos para valorizar, compreender, interpretar os dados empíricos, articulá-los com a teoria que fundamentou o estudo, além de outras leituras teóricas e interpretativas cuja necessidade foi dada pelos achados no trabalho de campo ${ }^{4}$.

Para análise de conteúdo foi utilizada a análise temática, como o próprio nome indica, o conceito central é o tema. Esse comporta um feixe de relações e pode ser graficamente apresentado através de uma palavra, uma frase, um resumo. Entende-se que o tema é a unidade de significação que se liberta naturalmente de um texto analisado segundo criterios relativos à teoria que serve de guia à leitura. Trabalhar com a análise temática, consistiu em descobrir os núcleos de sentido que fizeram parte da comunicação e cuja presença, ou frequência de aparição significou a emergência de discursos coerentes com o objetivo analítico escolhido 4 .

O processo de análise foi subdividido nos seguintes passos: (a) Organização dos dados, realizando a pré-análise, que consiste na organização, leitura e re-leitura do material, buscando coerência com os objetivos da pesquisa; (b) Classificação dos dados, explorando o material, realizando essencialmente a operação de codificação, fazendo recortes do texto em unidades de registro, uma frase, uma palavra ou um tema; (c) Análise propriamente dita em que os dados foram interpretados, colocando em evidencia as informações obtidas e fazendo inferências à luz da literatura existente ${ }^{4}$.

Ressalta-se que os sujeitos deste estudo foram doze docentes do curso de Graduação de Enfermagem de uma Instituição de Ensino Superior, privada, localizada na cidade do Rio de Janeiro, Brasil. Utilizou-se como critério de inclusão os docentes com mestrado e/ou doutorado.

A coleta dos dados foi realizada no local de trabalho dos sujeitos após o agendamento de horário de maio a junho 2012.

Os sujeitos da pesquisa assinaram o Termo de melhor conveniência para os mesmos, no período de Consentimento Livre e Esclarecido, autorizando sua participação na mesma conforme preconizado, além de ser garantido o sigilo e o anonimato dos mesmos, usando, portanto, o termo depoente, com vistas a respeitar os princípios éticos da pesquisa com seres humanos ${ }^{4,5}$.

Salienta-se ainda que o estudo em tela foi submetido à apreciação da Comissão de Ética e Pesquisa da Clínica Escola de Enfermagem da Universidade da Cidade, Unidade Praça Onze.

Não posso deixar de registrar a dificuldade com alguns docentes para realizar as entrevistas. Muitas vezes o horário marcado precisou ser trocado, uma vez que eles só podiam atender-me no horário de trabalho. Em outras oportunidades, a espera foi muito longa.

A despeito dos obstáculos, comuns a um estudo desta natureza a disposição da maioria para participar da pesquisa representou cooperação de valor inestimável para dar conta do objetivo desta investigação.

\section{Resultados, Análise e discusão}

Tendo por base a análise dos dados obtidos através das entrevistas, elaborou-se a primeira 
categoria intitulada "Contribuições da pesquisa para a prática baseada em evidências sob a percepção dos docentes" que deu origem a duas subcategorias temáticas envolvendo a mudança da prática, a qualidade da assistência e o desenvolvimento do corpo de conhecimento científico. A segunda categoria intitulada "Os desafios para a realização das práticas baseadas em evidências" comenta sobre a capacitação dos profissionais enfermeiros; o apoio das instituições prestadoras dos serviços de saúde no que diz respeito ao suporte organizacional; o direcionamento dos órgãos formadores para o preparo do enfermeiro frente à pesquisa, contribuindo dessa forma, com a fundamentação científica das suas ações para a realização da prática baseada em evidências.

Alguns países, numa tentativa de remediar o déficit de conhecimento e alcançar maior eficiência e efetividade na aplicação de recursos, iniciaram um movimento que assegurasse um cuidado baseado em pesquisas relevantes, especialmente aquelas articuladas ao tripé epidemiologia, bioestatística e informática para a melhoria da saúde da nação e que a utilização e desenvolvimento dessas pesquisas deveriam tornar-se parte integral dos serviços de saúde, onde o administrador, equipe médica, equipe de enfermagem e outros profissionais deveriam tomar decisões diárias baseadas nos resultados de investigações, nas experiências clínicas e habilidades, respeitando as preferências do paciente ${ }^{2,3}$.

A mudança da prática assistencial de enfermagem tem se configurado como uma necessidade na profissão para alcançar a qualidade da assistência, considerando que a enfermagem contemporânea requer dos profissionais atualizações dos conhecimentos, mecanismos e técnicas geradas pelas pesquisas que ofertam novas possibilidades de prevenção, diagnóstico e tratamento das doenças, visando à melhora da saúde humana ${ }^{6}$.

Diante do exposto, pode-se observar que sete entrevistados informaram que a prática baseada em evidências contribui na mudança da prática para melhorar a qualidade da assistência. Podemos exemplificar com as seguintes falas:

"[...] uma enfermagem moderna, dinâmica, com técnicas mais dinâmicas, lógico associada às atividades da ciência, de entendimento que vai fazer com que você consiga exercer a prática [...] consiga implementar uma nova prática [...]". (Depoimento 2)

\section{"[...] a prática baseada em evidências faz com que} seu cliente [...] consiga receber o melhor cuidado de enfermagem". (Depoimento 3)

"[...] falando das pesquisas devemos comentar que sem elas não teríamos fundamento teórico para realizar nossas atividades práticas e proporcionar uma melhor qualidade de atendimento a nossa clientela [...]". (Depoimento 6)

\section{“[...] uma prática baseada em evidências pode} modificar a prática, melhora a qualidade assistencial para ter uma resposta adequada [...] transforma a assistência padrão em diferenciada". (Depoimento 7)

\section{“[...] é importante que o profissional tenha em} mente que a pesquisa é fundamental para a transformação da prática, pois é através dela que ele vai atualizar seus conhecimentos, informações a respeito do que acontece em outras realidades. [...]" (Depoimento 9)

"[...] na assistência melhora sempre a qualidade do serviço que é prestado ao cliente [...] na minha visão vai melhorar a qualidade para o cliente e para a equipe de enfermagem, pois essa equipe vai trabalhar com mais segurança, sabendo o que realmente está fazendo [...]". (Depoimento 11 )

“[...] quando você faz pesquisa do que está vendo na prática comprova situações que podem mudar rotinas, protocolos [...]". (Depoimento 12) 
Em uma enfermagem moderna e dinâmica a pesquisa proporciona ao profissional o aprofundamento dos seus conhecimentos técnicos e científicos para a utilização de forma planejada e bem sustentada dos avançados protocolos e técnicas que servirão como base para seleção e organização dos cuidados inerentes ao paciente e proporcionar uma prática assistencial com qualidade ${ }^{2}$.

A mudança da prática assistencial conduz o profissional ao fazer reflexivo, a estar sempre buscando a melhoria da assistência prestada. Nesse sentido é importante ressaltar que a mudança dessa prática só acontecerá mediante o envolvimento de todos os profissionais no processo de transformação.

A satisfação das necessidades do paciente é o grau de excelência na qualidade assistencial, onde 0 cuidado alicerçado no conhecimento científico, agregado a experiência clínica do profissional e à visão crítica, proporciona resultados satisfatórios para ambas as partes (instituição e paciente), sendo a qualidade o fator de sobrevivência das instituições trazendo um diferencial competitivo das organizações de saúde em termos de serviços e proporcionando assim, não só a sobrevivência, mas o contínuo crescimento ${ }^{7}$.

Nesse processo, o enfermeiro é o profissional que coordena e gerencia toda a assistência a ser desenvolvida ao paciente e tudo o que o envolve no contexto da instituição hospitalar. O paciente e suas especificidades, suas necessidades, sua alta ou recuperação, constituem a principal razão da assistência de enfermagem, a qual deve, portanto, ser realizada eficientemente, com comprometimento de quem a desenvolve, garantindo qualidade do cuidado prestado e, principalmente, a satisfação do paciente e seus familiares 8 .

O enfermeiro elabora o levantamento de dados sobre o paciente, coleta, organiza e prioriza esses dados, estabelece o diagnóstico de enfermagem, desenvolve e implementa um plano de cuidado de enfermagem, e avalia aqueles cuidados em termos dos resultados alcançados pelo paciente. Em outras palavras, o enfermeiro utiliza o processo de enfermagem como metodologia assistencial para o planejamento e implementação dos cuidados de enfermagem 1 .

O processo de enfermagem é uma atividade deliberada, lógica e racional por meio da qual prática de enfermagem é desempenhada sistematicamente. Enquanto método para a organização da assistência essa metodologia compreende os cinco passos inter- relacionados: coleta de dados, diagnóstico, planejamento, implementação e avaliação. Assim, o processo de enfermagem é um método sistemático e organizado para ofertar cuidados de enfermagem individualizados, enfocando as respostas humanas de um a pessoa ou grupos a problemas de saúde reais ou potenciais. A realização dessa metodologia da assistência implica a prática baseada em evidências, tanto no que se referem aos dados coletados pelo enfermeiro (evidência baseada nas informações e nas condições clínicas do paciente) quanto ao julgamento clínico realizado por esse profissional para estabelecer os diagnósticos de enfermagem, planejar e avaliar as respostas à assistência implementada 1 .

O conhecimento adquirido através do processo de enfermagem sistematizado só tem sentido se for socializado e não permanecer encerrado em si mesmo. Uma vez que os resultados dessa sistematização da assistência unidos às pesquisas são determinados, devem ser disseminados de modo que a aplicação clínica ou a replicação por outros profissionais possam acontecer, pois as conclusões são fortalecidas e validadas por achados semelhantes em mais de um estudo de pesquisa².

Os discursos a seguir nos remetem ao evento fundamental para o desenvolvimento do corpo de conhecimento científico: 
"[...] você abre a internet e já vê a enfermagem ali [...]". (Depoimento 2)

"[...] que pode ser publicado e utilizado na prática [...]". (Depoimento 3)

"[...] em longo prazo, você pode ter estudos que comprovem a forma de fazer e desenvolver a prática assistencial, sendo uma fonte de divulgação [...]". (Depoimento 7)

\section{"[...] É importante que haja divulgação, que essas} pesquisas sejam publicadas, sejam apresentadas em eventos científicos, para que os outros profissionais tenham conhecimentos sobre os resultados [...]". (Depoimento 9)

\section{"[...] para mostrar a comunidade científica tem que} ser publicado primeiro, e segundo você tem que procurar espaço que são os eventos, palestras, cursos, mostrando que sua pesquisa dá certo [...]". (Depoimento 12)

Com a finalidade de promover a prática baseada em evidências é importante realizar maior disseminação das descobertas de pesquisas. Os enfermeiros pesquisadores devem divulgar internacionalmente tanto sua imagem quanto sua pesquisa, em organizações profissionais, órgãos de consumidores, agência do governo e empresas ${ }^{9}$.

Importante ressaltar que a internet e outros meios de comunicação eletrônica causam grande impacto na disseminação de informações de pesquisas a nível nacional e internacional, isso significa que as informações geradas podem ser visualizadas e utilizadas por profissionais de diversas áreas ${ }^{10}$.

A internet é uma ferramenta essencial para realização das práticas baseadas em evidências. Através dos portais da internet podemos acessar bancos de dados que contém dados préprimários, onde são publicadas informações que antecedem a publicação final dos resultados dos trabalhos científicos em andamento; dados primários que sevem primordialmente às atividades de pesquisa, elaboração de diretrizes ou à necessidade de informação mais recente para a aplicação clínica, e dados secundários utilizados em grande parte pelos profissionais que exercem atividades clínicas e precisam de informação confiável para cuidar da saúde das pessoas, solucionando diversos problemas da prática diária ${ }^{11}$.

Diante dos avanços tecnológicos significativos, atualmente, ainda há necessidade de ampliar o desenvolvimento da pesquisa no âmbito da enfermagem. Dentre outros desafios podemos ressaltar a capacitação desses profissionais, o poio das instituições prestadoras dos serviços de saúde no que respeita o suporte organizacional, o direcionamento dos órgãos formadores para o preparo do enfermeiro frente à pesquisa, possibilitando assim, a fundamentação científica das suas ações para a realização da prática baseada em evidências.

A PBE representa a ligação entre a pesquisa e a prática profissional, sendo um facilitador na capacitação e inserção do profissional no cotidiano do trabalho, permitindo a obtenção e a corroboração de conhecimentos.

Os enfermeiros são constantemente desafiados a produzir registros que sirvam como base para o desenvolvimento de futuras pesquisas, e com base nesses registros pode-se permanentemente construir melhores práticas assistências, além de implementar ações que visem melhoria nos resultados operacionais.

Para que o enfermeiro utilize pesquisas na prática, é preciso que desenvolva a habilidade de leitura que pode envolver: visita a banco de dados na busca de artigos que abordam problemas vivenciados na prática profissional; avaliação crítica dos estudos; após a leitura dos artigos, devem-se compartilhar as informações com outros colegas e promover reuniões para discutilas; participação em eventos onde pesquisas são 
apresentadas, discutidas, bem como a inserção em projetos de pesquisa ${ }^{12}$.

A utilização de pesquisa na enfermagem não deve permanecer apenas da dependência do enfermeiro, mas envolver também a organização em que este profissional está inserido, a qual deverá proporcionar condições de recursos humanos, físicos e financeiros compatíveis com a incorporação da inovação, da inserção e aplicação da pesquisa na prática para que possa acontecer essa transição de forma concisa e clara. Entretanto, não é o que se observa na grande maioria das instituições conforme evidenciado nas seguintes falas:

\section{"[...] muitos locais e instituições onde você não tem} os alicerces necessários para realizar a pesquisa [...]". (Depoimento 5)

"[...] instituições que tem interesse de investir na assistência dentro de uma atualidade realmente trazer a ciência à frente [...] nem todas as instituições estão abraçando esse tipo de causa [...]". (Depoimento 10).

Com esse entendimento as organizações deverão realizar a contratação de profissionais pesquisadores para auxiliar aos enfermeiros, por meio de programas de educação continuada, principalmente na orientação sobre a viabilidade de aplicação dos achados da pesquisa na prática assistencial contribuindo de forma efetiva para a aplicação das mesmas, proporcionando maior confiabilidade, credibilidade e organização para a implementação da prática baseada em evidências ${ }^{12}$.

Atualmente, devido a inúmeras inovações na área da saúde, a tomada de decisão dos enfermeiros necessita estar pautada em princípios científicos, ou seja, em pesquisas, a fim de selecionar a intervenção mais adequada para a situação específica de cuidado, uma vez que existem diferenças entre esperar que estes avanços tenham resultados positivos e verdadeiramente saber se eles funcionam, considerando o fato de que o paciente não pode esperar e precisa de um atendimento condizente com seu estado clínico ${ }^{13}$.

Destaca-se também a importância da existência de modelos colaborativos entre universidades e instituições de saúde no intuito de possibilitar a utilização instrumental da pesquisa na prática o que possibilitaria romper o vácuo existente entre o pesquisador/resultados das pesquisas e campo da prática ${ }^{14}$.

\section{Considerações finais}

Evidenciou-se que os sujeitos ao discorrerem acerca das contribuições da pesquisa para as práticas baseadas em evidências reconhecem sua importancia para a mudança da prática de enfermagem baseada no modelo tradicional para uma prática mais reflexiva alicerçada no conhecimento científico, promovendo assim, a melhoria da qualidade da assistência ao cliente, família e comunidade.

O desenvolvimento de pesquisas e a utilização de seus resultados na enfermagem encontram obstáculos que foram evidenciados nesse estudo, tais como: competência do enfermeiro para a aquisição de conhecimento, habilidades e valores essenciais à utilização de pesquisas e o suporte organizacional para proporcionar mecanismos facilitadores na utilização de pesquisas no ambiente de trabalho.

Os enfermeiros tendo em mãos a melhor informação científica disponível, aliado à sua experiencia profissional e adequação à situação clínica poderão decidir pela melhor evidência que atenda as necessidades reais de seus clientes, contribuindo para reduzir as chances de desfechos negativos, pois estará realizando a prática diária através do conhecimento e intervenções que funcionam, aumentando a eficiência profissional com menor desperdício de recursos e energia tanto do profissional quanto do paciente sobre seu cuidado. 
Com a finalidade de promover mudanças nas atitudes do enfermeiro frente à pesquisa, esse processo deve ter início na graduação por meio de inclusão de disciplinas curriculares, que busquem desenvolver nos estudantes a compreensão da pesquisa para a enfermagem, do processo de pesquisar e da utilização assistência prestada ao cliente. Os desenvolvimentos dos resultados de pesquisas para a melhoria da de habilidades para a avaliação crítica de estudos na graduação auxiliará o enfermeiro na tomada de decisão sobre quais são os resultados de pesquisas Em síntese, para as práticas baseadas em evidencias tornar-se realidade, são necessárias mudanças nas esferas educacional, organizacional e individual. adequados para o uso na sua área de atuação.

\section{Referências bibliográficas}

1. Galvão CM, Sawada NO, Rossi LA. A prática baseada em evidências: considerações teóricas para sua implementação na enfermagem perioperatória. Rev Latino Am Enfermagem 2002 Set/Out; 10(5):690-5.

2. Craven RF, Hirnle CJ. Fundamentos de enfermagem, saúde e função humanas. 4. ed. Rio de Janeiro: Guanabara Koogan; 2006.

3. Potter PA, Perry AG. Fundamentos de enfermagem. 7. ed. Rio de Janeiro: Elsevier; 2009.

4. Minayo MCS. Pesquisa social: teoria, método e criatividade. 21. ed. Petrópolis, RJ: Vozes; 2008.

5. Ministério da Saúde (Br). Resolução 196/96 do Conselho Nacional de Saúde/MS sobre diretrizes e Normas Regulamentadoras de Pesquisa envolvendo seres humanos. Diário Oficial da União, Brasília(DF); 10 out 1996.
6. Lacerda RA, Nunes BK, Batista AO, Egry EY, Graziano KU, Angelo M, et al. Práticas baseadas em evidências publicadas no Brasil: identificação e análise de suas vertentes e abordagens metodológicas. Rev Esc Enferm USP 2011 Jun;45(3):777-86.

7. Oguisso T. Ética e bioética: desafios para a enfermagem e a saúde. Barueri, SP: Manole; 2006.

8. Paladini EP, Bouer G, Ferreira JJA, Carvalho MM, Miguel PAC, Samohyl RW, et al. Gestão de qualidade: teoria e casos. Rio de Janeiro: Elsevier; 2006.

9. Barbosa LR, Melo MRAC. Relações entre qualidade da assistência de enfermagem: revisão integrativa da literatura. Rev Bras Enferm 2008 Maio/Jun;61(2):366-70.

10.Carvalho EC. A produção do conhecimento em enfermagem. Rev Latino Am Enfermagem 1998 Jan;6(1):119-22.

11.Polit DF, Beck CT, Hungler BP. Fundamentos de pesquisa em enfermagem: métodos, avaliação e utilização. 5. ed. Porto Alegre: Artmed; 2004.

12.Galvão CM, Sawada NO. Prática baseada em evidências: estratégias para sua implementação na enfermagem. Rev Bras Enferm 2003;56(1):57-60.

13.Pedrolo E, Danski MTR, Mingorance P, Lazzari LSM, Méier MJ, Crozeta K. A prática baseada em evidências como ferramenta para a prática profissional do enfermeiro. Cogitare Enferm 2009 Out/Dez; 14(4):760-3.

14.Padilha MI. Pesquisa translacional: qual a importância para a prática da enfermagem? Texto Contexto Enferm 2011 Jul/Set;20(3):419-20. 